\title{
ANÁLISE DA ESTRUTURA DE MERCADO NA CADEIA PRODUTIVA DO LEITE NO PERÍODO DE 1998 A 2008
}

\author{
Fabiano Luiz Alves Barros ${ }^{2}$ \\ João Ricardo Ferreira de Lima ${ }^{3}$ \\ Rosangela Aparecida Soares Fernandes ${ }^{4}$
}

\begin{abstract}
Resumo: A cadeia produtiva do leite figura entre os produtos mais importantes da agropecuária nacional, sendo responsável por uma parcela significativa do agronegócio. Nos últimos anos o setor tem passado por diversas transformações em sua estrutura, com o aumento na participação de mercado das empresas relativamente menores e redução na parcela de mercado das maiores empresas. O problema de pesquisa deste trabalho é entender a dinâmica entre os desempenhos da indústria e do varejo e o efeito sobre os preços pagos aos produtores de leite. Especificamente se busca calcular os índices de concentração no setor e de causalidade entre os preços recebidos pelo produtor, markup da indústria e do varejo. A partir de séries temporais de preço é estimado um modelo VAR e realizado posteriormente o teste de causalidade de Granger. Os resultados reforçam a tese de desconcentração no setor, e os testes de causalidade indicam baixo poder de oligopsônio do varejo e da indústria.
\end{abstract}

Palavras-chaves: Concentração de mercado, leite, causalidade de Granger.

Recebido em: 18/02/2010; Aceito em: 09/08/2010.

2 Graduado em Economia pela Universidade Federal de Campina Grande - UFCG. Email: fabianowar@ hotmail.com Doutor em Economia Aplicada/DER-UFV. Prof. Adjunto do Departamento de Ciências Fundamentais e Sociais do Centro de Ciências Agrárias da UFPB. Pesquisador do LAPEA - Laboratório de Pesquisas em Economia Aplicada/UFCG. Email: jricardo@cca.ufpb.br

4 Doutoranda em Economia Aplicada/DER-UFV. Profa. Assistente da Universidade Federal Rural do Rio de Janeiro- UFRRJ. Email: roaeconomista@yahoo.com.br 


\section{Introdução}

A cadeia produtiva do leite está entre as mais importantes da agropecuária nacional, sendo responsável por uma parcela significativa do agronegócio. A cada $\mathrm{R} \$ 1,00$ de aumento da produção no sistema agroindustrial do leite, tem-se uma elevação de, aproximadamente, $\mathrm{R} \$ 5,00$ no Produto Interno Bruto (PIB), o que faz com que o produto esteja à frente de outros setores do agronegócio brasileiro (ALVIM et al., 2002).

Quanto ao mercado internacional, nos últimos anos, a adoção de técnicas mais sofisticadas viabilizou o aumento da captação de leite in natura. Além disso, a expansão da demanda de leite e derivados tem também contribuído para o crescimento da produção leiteira.

Quando se faz a distribuição da produção de leite no mercado internacional, observa-se, na Tabela 1, que os Estados Unidos ocupam a primeira posição no ranking dos maiores produtores do mundo, com uma média de $15 \%$ da produção mundial e expansão da produção acumulada, da ordem de 15,02\%. Em seguida, está a Índia, com 7,5\% do total. A China ocupa a terceira posição, com 5,86\%. Em quarto lugar, está a Federação Russa, com, aproximadamente, $5,7 \%$. O Brasil é o sexto maior produtor mundial de leite, com participação de $4,52 \%$. 
Fabiano Luiz Alves Barros, João Ricardo Ferreira de Lima \& Rosangela Aparecida Soares Fernandes

Tabela 1 - Principais países produtores de leite (mil/toneladas), 2007

\begin{tabular}{|c|c|c|c|c|}
\hline Rank & Países & $\begin{array}{l}\text { Produção de leite } \\
\text { (mil ton.) }\end{array}$ & $\begin{array}{l}\text { Percentual } \\
\text { total }\end{array}$ & $\begin{array}{l}\text { Percentual } \\
\text { acumulado }\end{array}$ \\
\hline $1^{\circ}$ & $\begin{array}{l}\text { Estados } \\
\text { Unidos }\end{array}$ & 84.189 & 15,02 & 15,02 \\
\hline $2^{\circ}$ & Índia & 42.140 & 7,52 & 22,54 \\
\hline $3^{\circ}$ & China & 32.820 & 5,86 & 28,39 \\
\hline $4^{\circ}$ & $\begin{array}{l}\text { Federação } \\
\text { Russa }\end{array}$ & 31.950 & 5,70 & 34,09 \\
\hline $5^{\circ}$ & Alemanha & 27.900 & 4,98 & 39,07 \\
\hline $6^{\circ}$ & Brasil & 25.327 & 4,52 & 43,59 \\
\hline
\end{tabular}

Fonte: Embrapa Gado de Leite, 2009.

A estrutura brasileira de mercado pode ser chamada de atomizada, pois sua produção se encontra pulverizada com a participação de pequenos, médios e grandes produtores. Tal fato não favorece a disseminação de informações, elevando assim os custos de captação, armazenamento. Segundo Campos e Piacenti (2007), a produção brasileira de leite está concentrada nos estados de Minas Gerais, Goiás, Paraná, Rio Grande do Sul e São Paulo.

Além disso, a estrutura organizacional da indústria de laticínios sofreu transformações nos últimos anos. Verifica-se um aumento na participação de mercado das empresas relativamente menores, caracterizado especialmente por elevações na captação de leite destinado ao processamento e, como consequência, as empresas de maior porte apresentaram queda nas parcelas de mercado e diminuição em suas receitas. Diante desse cenário, surge o problema de pesquisa deste trabalho: qual a dinâmica entre os desempenhos da indústria e do varejo e o efeito sobre os preços pagos aos produtores de leite UHT de São Paulo? A escolha por esse estado deve-se à sua forte representação no mercado interno, sendo ainda o maior mercado consumidor do produto (FERNANDES e SANTOS, 2009). A hipótese da pesquisa é que a 
desconcentração no setor reduz o poder de oligopsônio da indústria, que é menos forte no varejo.

O trabalho está dividido em quatro partes, além da introdução. $\mathrm{Na}$ primeira, é feita a fundamentação teórica do estudo na qual será destacado o Paradigma Estrutura-Conduta-Desempenho. Na segunda parte, é apresentada a metodologia enfatizando os procedimentos econométricos utilizados. Em seguida, são relatados os índices de concentração industrial e os resultados do modelo econométrico adotado. Por fim, são apresentadas as conclusões do estudo.

\section{Referencial Teórico}

No campo da organização industrial, busca-se estudar as inter-relações das políticas das unidades produtivas em relação às demais firmas e sua relação com o mercado consumidor. Desta maneira, são definidos dois tipos de vertentes teóricas: uma embasada na Escola de Chicago e a outra no modelo de Estrutura-Conduta-Desempenho (ECD). Segundo Fernandes (2006), o modelo ECD afirma que qualquer forma de obstáculo à entrada de novas empresas aumenta o poder de mercado no setor. $\mathrm{O}$ paradigma ECD busca explicar, de forma linear, como a estrutura de um setor, que depende de determinadas condições como tecnologia e demanda, afeta sua conduta, que, por sua vez, determina o desempenho das firmas. Segundo Martin (1993), a estrutura das firmas no mercado mostra como são tomadas as decisões (conduta) que, por conseguinte, modificam seu nível de eficiência (desempenho).

De acordo com a teoria microeconômica, existem diversas estruturas de mercado. A concorrência perfeita é uma estrutura que apresenta um grande número de vendedores e compradores e, assim sendo, a ação de uma empresa ou consumidor não afeta o preço, pois suas produções são mínimas frente ao total produzido. As barreiras na concorrência perfeita são inexistentes, podem entrar ou sair do mercado sem ônus. Para maximizar seu lucro, a firma deve escolher um nível de produção que 
iguale a receita marginal ao custo marginal, no ramo crescente da curva deste.

Em outro extremo está o monopólio, representando uma única empresa dominando a oferta de produtos ou serviços, que não têm substitutos próximos. Segundo Varian (2006), o monopolista reconhece sua influência sobre o preço do mercado e escolhe o nível de preço e de produção que maximize seus lucros totais. As barreiras à entrada de novas empresas nesse mercado são bastante fortes, já que o monopolista pode modificar seu nível de produção ou até mesmo o preço por ser o único ofertante no mercado. Os preços no monopólio são mais elevados do que os considerados na concorrência perfeita. O monopsônio é um tipo de estrutura existente pelo lado da demanda, caracterizando-se como único comprador de matéria-prima ou produto primário.

O oligopólio, por sua vez, caracteriza-se pelo número reduzido de firmas dentro do mercado e pelo reconhecimento de sua interdependência mútua. Dessa forma, cada uma das empresas pode influenciar seu preço de mercado ao variar sua produção. O inverso não é verdadeiro, pois cada firma não é grande o suficiente para atuar como monopolista dentro do mercado. A interdependência entre as firmas, isto é, o poder de ação de uma delas influenciar o preço ou a parcela de mercado das outras, faz com que cada uma leve em conta as ações e reações de empresas terceiras (MARTIN, 1993). Essa estrutura de mercado possui barreiras à entrada, pois as economias de escalas, as patentes e a intensidade de capital configuram impedimentos para a chegada de novas firmas. Assim, os agentes econômicos que agem nessa estrutura podem exercer poder de mercado.

O paradigma ECD admite que quanto mais a estrutura do mercado se distancia da competição perfeita, mais provável é o exercício de poder de mercado. Todavia, quando o mercado se aproxima da estrutura de concorrência perfeita, havendo um elevado número de empresas, produtos homogêneos e liberdade de entrada, as firmas possuem poucas estratégias disponíveis, além de buscar reduzir os custos. Esse mercado 
tem bom desempenho quando produz uma quantidade próxima à da concorrência perfeita (CARLTON \& PERLOFF, 2005).

A conduta pode ser caracterizada como um conjunto de estratégias que as firmas podem adotar dentro de um mercado. Para Martin (1993), a conduta de mercado só se torna relevante quando esse é imperfeito, já que em mercados de concorrência pura não se adotam comportamentos estratégicos. Sendo assim, as principais formas de conduta são: cooperação, comportamento estratégico, propaganda, pesquisa e desenvolvimento.

O desempenho consiste no grau de bem-estar social gerado pelas atividades desenvolvidas no mercado; pode ser considerado em termos de eficiência, lucratividade e progressividade (FERNANDES, 2006). A firma que conseguir oferecer benefícios para os consumidores terá sucesso.

\subsection{Referencial Analítico}

Da mesma forma que Muller et al. (2006), a análise não será feita com dados do tipo cross-section, mas por meio de uma série temporal. Dado que a abordagem da ECD admite efeitos dinâmicos, são construídas duas medidas de desempenho (markups), uma para a indústria e outra para o varejo, além dos preços pagos ao produtor no período de setembro de 1998 a dezembro de 2008, como demonstrado em tópico posterior sobre a fonte de dados.

\subsubsection{Teste de raiz unitária}

O Processo Gerador de Dados (PGD) de uma série temporal pode ser dividido em estacionário e não estacionário. O PGD é estacionário quando 
sua média e variância são constantes no tempo e a sua covariância entre dois períodos de tempo depende de defasagens eles, conforme as equações abaixo:

$\varepsilon\left(Y_{t}\right)=\mu$

$V\left(Y_{t}\right)=\sigma^{2}$

$\operatorname{Cov}\left(Y_{t}, Y_{s}\right)=f(k) ; t \neq s$

sendo k o número de defasagens entre dois períodos.

Os problemas relativos às séries não estacionárias referem-se à limitação para previsões. Por outro lado, é possível encontrar alguma correlação estatística entre as variáveis devido a sua tendência, sendo esta uma relação espúria ${ }^{5}$. Segundo Gujarati (2006), a estacionariedade pode ser analisada a partir do teste de correlograma, que corresponde a um gráfico de autocorrelação para diferentes defasagens. Se a série diminui rapidamente, essa pode ser estacionária. Para uma análise mais formal, utiliza-se o teste de raiz unitária; neste trabalho, o utilizado será DickeyFuller-Mínimos Quadrados Generalizados (DF-GLS).

O DF-GLS é uma versão modificada do Teste de Dickey-Fuller Aumentado (ADF), sendo uma verificação de raiz unitária de segunda geração, que possui maior potência. Existem duas hipóteses alternativas: $\mathrm{y}_{\mathrm{t}}$ é estacionário sobre uma tendência linear ou $\mathrm{y}_{\mathrm{t}}$ é estacionário sem a tendência linear. Considerando a primeira, o teste DF-GLS é realizado inicialmente estimando o intercepto e a tendência via Mínimos Quadrados Generalizados (MQG), cuja estimação por MQG é feita pela inclusão de novas variáveis $\tilde{y}_{\mathrm{t}}, \mathrm{x}_{\mathrm{t}}$ e $\mathrm{z}_{\mathrm{t}}$, em que

Para Gujarati (2006, p. 646), “[...] regressão espúria é uma regressão sem sentido”. 


$$
\begin{aligned}
& \tilde{y}_{1}=\mathrm{y}_{1} \\
& \tilde{y}_{\mathrm{t}}=\mathrm{y}_{\mathrm{t}}-\alpha^{*} y_{t-1} \mathrm{t}=2, \ldots, \mathrm{T} \\
& x_{1}=1 \\
& x_{t}=1-\alpha^{*} \mathrm{t}=2, \ldots, \mathrm{T} \\
& z_{1}=1 \\
& z_{t}=t-\alpha^{*}(t-1) \\
& \mathrm{e} \\
& \alpha^{*}=1-(13.5 / T)
\end{aligned}
$$

Faz-se então uma regressão por Mínimos Quadrados Ordinários:

$$
\tilde{y}_{t}=\delta_{0} x_{t}+\delta_{1} z_{t}+\epsilon_{t}
$$

em que os estimadores $\hat{\delta}_{0} e \hat{\delta}_{1}$ são usados para retirar a tendência de $\mathrm{y}_{\mathrm{t}}$. O passo seguinte é gerar $\mathrm{y}^{*}$

$y_{t}^{*}=y_{t}-\left(\hat{\delta}_{0}+\hat{\delta}_{1} t\right)$

Finalmente, o teste DF-GLS envolve a estimação do ADF, com a substituição das variáveis do GLS modificada por $y_{t}^{*}$ e uma regressão por MQO

$$
\begin{aligned}
& \Delta y_{t}^{*}=\alpha+\beta y_{t-1}^{*}+\sum_{j=1}^{k} \varsigma \Delta y_{t-j}^{*}+\epsilon_{t} \\
& \text { e, então, testar a hipótese nula de } \beta=0 .
\end{aligned}
$$


Com relação à segunda hipótese alternativa, o procedimento é semelhante, mas define-se $\alpha^{*}=1-(7 / T)$, eliminando $\mathrm{z}$ da regressão por MQG, calculando $y_{t}^{*}=y_{t}-\widehat{\delta}_{0}$ e estimando a regressão do ADF sobre as novas variáveis transformadas. A hipótese nula do teste é $\beta=0$. Para outras informações, pode-se consultar o manual TS do software Stata 11.

\subsubsection{O Teste de Causalidade de Granger após o VAR}

Esta pesquisa difere, metodologicamente, dos trabalhos de Muller et al. (2006) e Fernandes (2006). No primeiro, os autores estimam um modelo Vetorial Autorregressivo Estrutural (SVAR) buscando incorporar na análise as relações contemporâneas. No segundo, encontram-se diversos testes de causalidade de Granger não multivariados e, assim, não realizados após a estimação de um modelo Vetor Autorregressivo (VAR). Nesta pesquisa, a análise do poder de oligopsônio da indústria e do varejo é realizada por meio de testes de Causalidade de Granger após a estimação do modelo VAR (um para cada equação).

O modelo VAR tem por premissa a utilização de algumas variáveis endógenas em conjunto, de forma que cada uma é explicada pelo seu valor defasado (excedido) e, também, pelos valores defasados das outras variáveis que compõem o modelo. Algebricamente, um VAR de ordem $\mathrm{p}, \operatorname{VAR}(\mathrm{p})$, sem variáveis exógenas, é dado por

$y_{t}=v+A_{1} y_{t-1}+\ldots+A_{p} y_{t-p}+u_{t}$

em que $y_{t}=\left(y_{1 t}, \ldots, y_{K t}\right)^{\prime}$ é um vetor de variáveis $\mathrm{Kx} 1 ; \mathrm{A}_{1}$ até $\mathrm{A}_{\mathrm{p}}$ são matrizes $\mathrm{KxK}$ de parâmetros; v é um vetor $\mathrm{Kx} 1$ de parâmetros; e, $u_{t}$ é um resíduo ruído branco com média zero, sem autocorrelação e com matriz de variância-covariância igual a $\Sigma$. Um VAR pode ser visto como a forma reduzida de um sistema de equações simultâneas dinâmico. 
Um modelo VAR é considerado estável quando estacionário. A estabilidade está relacionada ao fato de que o efeito de choques eventuais desaparece ao longo do tempo, ou seja, o sistema volta ao equilíbrio. Considere-se um VAR com 1 lag, VAR(1)

$$
\begin{aligned}
& y_{t}=v+\mathrm{A}_{1} y_{t-1}+u_{t} \\
& y_{t}=v+\mathrm{A}_{1} L y_{t}+u_{t} \\
& y_{t}-\mathrm{A}_{1} L y_{t}=v+u_{t} \\
& {\left[I-\mathrm{A}_{1} \mathrm{~L}\right]=0}
\end{aligned}
$$

Todas as raízes do polinômio devem ser $>1$ em valor absoluto, sendo o número das raízes dado por p.k. A definição do número de lags do VAR é feita com base nos critérios de informações Akaike, Schwars, Hannan Quinn, e, na dúvida, busca-se utilizar o critério da parcimônia, isto é, o menor número de lags. As análises feitas com VAR são previsões, funções impulso-resposta, ou seja, a resposta do sistema a choques nas variáveis através dos erros e o teste de causalidade de Granger.

O teste de causalidade desenvolvido por Granger tem por definição evidenciar se uma variável causa a Granger outra variável. Uma variável $z$ é dita que causa a Granger uma variável x se, dados os valores passados de $\mathrm{x}$, os valores passados de $\mathrm{z}$ são úteis para prever $x$.

Para estimação, tem-se:

$$
\begin{aligned}
& z=\sum_{i=1}^{n} \alpha_{i} x_{t-1}+\sum_{j=1}^{n} \beta_{j} z_{t-1}+u_{1 t} \\
& x=\sum_{i=1}^{n} \lambda_{i} x_{t-1}+\sum_{j=1}^{n} \delta_{j} z_{t-1}+u_{2 t}
\end{aligned}
$$

sendo que os distúrbios $u_{1 t}$ e $u_{2 t}$ não estão correlacionados. Se a análise busca verificar a relação de causalidade entre múltiplas variáveis, essa 
deve ser feita após a estimação do modelo VAR. Na regressão dada em (12), testa-se a hipótese nula de que os alfas estimados da variável $\mathrm{x}$ defasada são conjuntamente iguais a zero. Não rejeitar essa possibilidade é equivalente a não recusar a hipótese de que a variável $\mathrm{x}$ não causa a Granger a variável z. No teste de Granger, após o VAR, para cada uma de suas equações, testa-se a hipótese de que cada uma das demais variáveis endógenas não causa a Granger a variável dependente na respectiva equação.

\subsection{Fonte dos Dados}

Os dados utilizados neste trabalho foram obtidos no Instituto de Economia Agrícola (IEA), $\operatorname{S} \operatorname{tot}^{6}$ Consultoria LTDA., Departamento Intersindical de Estatística e Estudos Socioeconômicos (Dieese) e Embrapa Gado de Leite. Além de considerar os preços pagos ao produtor, o problema de pesquisa deste trabalho gera a necessidade de criar as variáveis do markup relativas ao varejo (representadas pela diferença entre os preços arrecadados no varejo e os recebidos pela indústria) e o markup relativo à indústria, representado pela diferença entre o preço recebido por esta e o pago aos produtores, de acordo com Muller et al. (2006), como demonstrado nas equações (14) e (15):

$M k_{v}=\frac{p_{v}-P_{i}}{p_{v}}$

em que $\mathrm{Pv}=$ Preço do Varejo e Pi $=$ Preço da Indústria

$M K_{i}=\frac{p_{i}-P_{p}}{P_{p}}$

em que $\mathrm{Pi}=$ Preço da Indústria e $\mathrm{Pp}=$ Preço recebido pelo produtor.

\footnotetext{
Os autores agradecem em especial à Scot Consultoria que forneceu os dados a um custo reduzido, ao saber que as informações destinavam-se exclusivamente a um estudo acadêmico.
} 
Os dados aqui apresentados foram deflacionados pelo IGP-DI, tomando por referência ago/2009.

\section{Resultados}

\subsection{Concentração de Mercado}

A Tabela 2 reporta a evolução da estrutura industrial no mercado lácteo brasileiro no período de 1998 a 2007. As parcelas de mercado de cada uma das quatro maiores empresas da indústria láctea foram obtidas pela razão entre a recepção de leite e a produção total do Brasil. Com o objetivo de se obter o grau de concentração de tais organizações, as parcelas de mercados foram agrupadas conforme o índice de concentração CR4.

Verifica-se que, ao longo do período em análise, a Nestlé perdeu 2,34\% da participação no mercado ao ano; no entanto, ainda permanece com a posição de empresa líder. A Itambé também perdeu parcela significativa de mercado, de $1,93 \%$ a.a. A Elegê, porém manteve sua participação praticamente estável no setor. Assim, não se encontrou nas estimativas uma tendência definida e a taxa de crescimento calculada não foi estatisticamente significativa. A Parmalat foi a empresa que mais perdeu parcela de mercado, como demonstra o cálculo da taxa de crescimento de sua participação no setor (de $-9,5 \%$ a.a). A crise dessa organização em 2004 pode explicar sua mudanca de posicionamento, que caiu da segunda posição para a quarta no nível de participação no mercado. Segundo Fernandes (2006), a empresa vinha passando por problemas associados a um padrão de crescimento desordenado caracterizado por compras e fechamentos de diversos laticínios em todo o Brasil, que se manifestava desde 1998, sendo que o maior dano aconteceu em dezembro de 2003, quando a Parmalat passou por uma crise financeira em sua matriz, que levou ao fechamento de suas unidades produtivas. 
Fabiano Luiz, Alves Barros, João Ricardo Ferreira de Lima \& Rosangela Aparecida Soares Fernandes

Tabela 2 - Participação da recepção de leite (\%) e índices de concentração em porcentagem, Brasil, 1998 - 2007

\begin{tabular}{l|cccccccccccc|c|c}
\hline Empresa & 1998 & 1999 & 2000 & 2001 & 2002 & 2003 & 2004 & 2005 & 2006 & $2007^{\mathrm{a}}$ & $\mathrm{TC}^{\mathrm{b}}$ \\
\hline Nestlé & 30 & 29 & 28 & 27 & 29 & 29 & 29 & 28 & 24 & 22 & $-2,34 * *$ \\
Elege & 13 & 14 & 15 & 15 & 14 & 13 & 14 & 14 & 13 & 16 & 0,44 \\
Itambé & 17 & 17 & 16 & 16 & 14 & 15 & 16 & 16 & 15 & 13 & $-1,93 * *$ \\
Parmalat & 18 & 17 & 19 & 18 & 15 & 13 & 8 & 10 & 9 & 9 & $-9,50 * * *$ \\
Outras & 22 & 23 & 22 & 24 & 29 & 30 & 33 & 32 & 39 & 40 & $7,50 * * *$ \\
CR4 & 0,78 & 0,77 & 0,78 & 0,76 & 0,71 & 0,70 & 0,67 & 0,68 & 0,61 & 0,60 & $-3,02 * * *$ \\
\hline
\end{tabular}

${ }^{\mathrm{a}}$ Os dados da produção de 2007, divulgados pela Embrapa Gado de Leite, são resultados preliminares.

${ }^{\mathrm{bb}}$ Foram calculadas as taxa geométricas de crescimento (TC), no período de 1998 a 2007, pelos autores.

***, ** e * representam, respectivamente, significância de $1 \%, 5 \%$ e $10 \%$.

Fonte: Embrapa Gado de Leite, (2009).

Na Tabela 2, a taxa geométrica de crescimento, de mais de 7,50\% ao ano, para o grupo Outras, formado por pequenas empresas do setor, é bastante significativa estatisticamente. A crise pode ter criado oportunidade para a intensificação do processo de crescimento de empresas relativamente menores, uma vez que os produtores e cooperativas, diretamente ligados à Parmalat, em sua maioria, transferiram sua produção para outras organizações concorrentes que, aos poucos, ocupam o espaço deixado pela empresa. Em especial, destaca-se a redução da participação da Parmalat no segmento de leite longa vida, no qual era líder. Como consequência, novas marcas regionais passaram a surgir nas gôndolas dos supermercados. Analisando o índice de concentração CR4, percebe-se que houve uma diminuição na taxa geométrica de crescimento de mais de $3 \%$ ao ano, com redução de 0,78 para 0,60 , indicando desconcentração no setor. 


\subsection{VAR e Teste de Causalidade de Granger}

Inicialmente, é necessário visualizar o gráfico das séries para ter uma ideia de seu comportamento no tempo. No eixo das abscissas, estão o nível de preço e os markups, enquanto o eixo das ordenadas representa os anos de 1998 a 2008. Ao longo de todo o período, os preços do markup da indústria sofreram uma variação negativa. Com relação ao comportamento do markup do varejo, este também sofreu uma variação negativa nos anos estudados, ao contrario dos preços dos produtores que apresentaram um comportamento ascendente, conforme Figura 1.

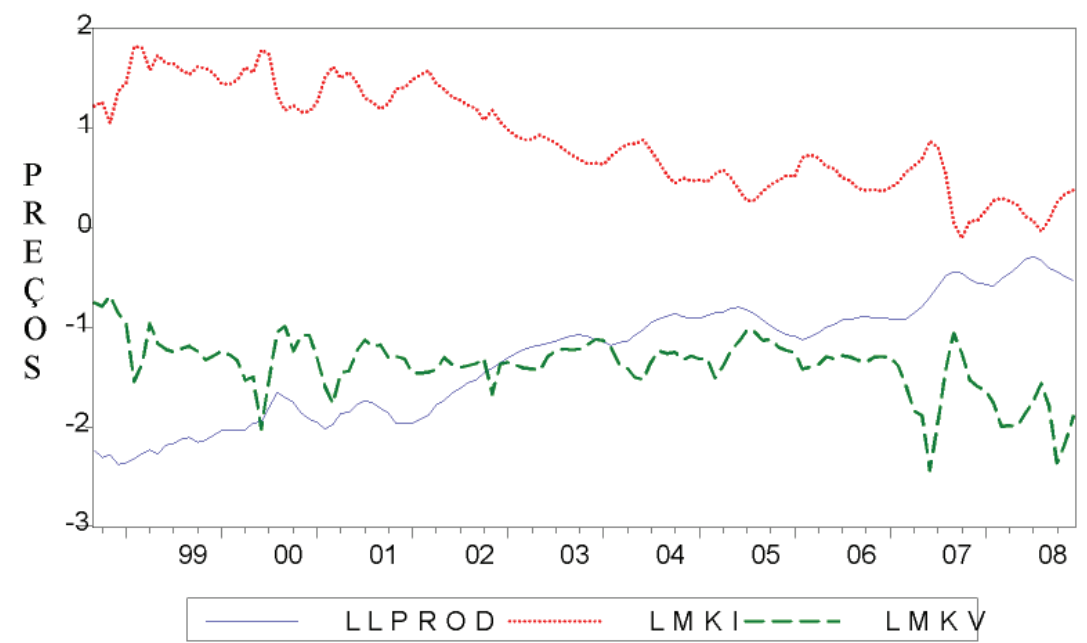

Figura 1 - Comportamentos dos preços, Logaritmo dos Preços ao Produtor (LLPROD), Logaritmo do Markup da Indústria (LMKI) e Logaritmo do Markup do Varejo (LMKV).

Fonte: Dados da pesquisa. 
O passo seguinte é analisar a estacionariedade das séries, realizando o teste de raiz unitária DF-GLS, considerando as variáveis em nível e a estacionariedade sobre uma tendência linear. Os resultados estão apresentados na Tabela 3 demonstram, para as três séries, que o valor calculado é maior do que o crítico, considerando 5\% de significância estatística. Para as três variáveis, rejeita-se a hipótese nula de que a série é um passeio aleatório, possivelmente com drift, pois as variáveis são estacionárias em nível, sendo então I(0).

Tabela 3 - Testes de raízes unitárias DF-GLS para as variáveis LLPROD, LMKI e LMKV

\begin{tabular}{c|c|c|c|c}
\hline Séries & Defasagens & $\begin{array}{c}\text { Equação de } \\
\text { teste }\end{array}$ & Estatística t & $\begin{array}{c}\text { Valor } \\
\text { critico(5\%) }\end{array}$ \\
\hline LPROD & 1 & $\begin{array}{c}\text { Intercepto e } \\
\text { tendência }\end{array}$ & $-3,152$ & $-3,008$ \\
LMKI & 1 & $\begin{array}{c}\text { Intercepto e } \\
\text { tendência }\end{array}$ & $-3,279$ & $-3,008$ \\
LMKV & 1 & $\begin{array}{c}\text { Intercepto e } \\
\text { tendência }\end{array}$ & $-4,392$ & $-3,008$ \\
\hline
\end{tabular}

Fonte: Dados da pesquisa.

Dado que as séries são todas integradas de ordem zero, deve-se estimar um modelo VAR. Assim, a etapa seguinte do trabalho é definir o número de lags do VAR. Isso pode ser determinado por meio dos critérios de Hannan-Quinn, Akaike e Schwarz. Como mostrado na Tabela 4, a minimização dos erros de Predição Final, a sequência modificada de LR ao nível de $5 \%$ e o critério de Akaike apontam para quatro defasagens, ao passo que o critério Hannan-Quinn e o Schwarz apontam para duas defasagens. Dados os resultados, optou-se pela primeira norma. 
Tabela 4 - Escolha das defasagens para o VAR

\begin{tabular}{ccc|c|c|c|c|c}
\hline Lag & LogL & LR & FPE & AIC & SC & HQ \\
\hline 0 & -11.12024 & NA & 0.000256 & 0.243452 & 0.314666 & 0.272361 \\
1 & 469.0397 & 927.2055 & $7.59 \mathrm{e}-08$ & -7.879996 & -7.595141 & -7.764361 \\
2 & 501.6546 & 61.29337 & $5.05 \mathrm{e}-08$ & -8.287148 & $\mathbf{- 7 . 7 8 8 6 5 3 ^ { * }}$ & $\mathbf{- 8 . 0 8 4 7 8 7 ^ { * }}$ \\
3 & 510.5564 & 16.26879 & $5.07 \mathrm{e}-08$ & -8.285454 & -7.573319 & -7.996368 \\
4 & 524.4417 & $\mathbf{2 4 . 6 5 8 4 5}$ & $\mathbf{4 . 6 7 e - 0 8 ^ { * }}$ & $\mathbf{- 8 . 3 6 9 6 8 4 ^ { * }}$ & -7.443908 & -7.993872 \\
5 & 530.6911 & 10.77478 & $4.90 \mathrm{e}-08$ & -8.322260 & -7.182843 & -7.859722 \\
6 & 536.5059 & 9.724737 & $5.20 \mathrm{e}-08$ & -8.267342 & -6.914285 & -7.718078 \\
7 & 544.7731 & 13.39857 & $5.29 \mathrm{e}-08$ & -8.254708 & -6.688010 & -7.618718 \\
8 & 551.6745 & 10.82807 & $5.52 \mathrm{e}-08$ & -8.218525 & -6.438187 & -7.495810 \\
\hline
\end{tabular}

* indica o número de lags a ser escolhido.

LR: Sequência modificada de LR, teste estatístico ao nível de 5\%;

FPE: Erro de predição final;

AIC: Critério de Akaike;

SC: Critério de Schwarz;

HQ: Critério de Hannan-Quinn.

Fonte: Dados da pesquisa.

Na sequência, faz-se necessário estimar o VAR. Na Tabela 5, estão os preços pagos aos produtores, influenciados pelo markup da indústria na primeira defasagem. O markup da indústria também é influenciado de forma expressiva pelo do varejo na primeira defasagem, assim como os preços pagos aos produtores. Já em relação ao markup do varejo, este passa a ser influenciado pelo da indústria na primeira defasagem e por si próprio. O R ${ }^{2}$ para LLPROD, LMKI e LMKV foi de, respectivamente, $0,9973,0,9781$ e 0,6978 . 
Fabiano Luiz Alves Barros, João Ricardo Ferreira de Lima \& Rosangela Aparecida Soares Fernandes

Tabela 5 - Modelo VAR estimado

\begin{tabular}{|c|c|c|c|c|c|c|c|}
\hline & LLPROD & LMKI & LMKV & & LLPROD & LMKI & LMKV \\
\hline \multirow[t]{2}{*}{ LPROD(-1) } & $1.521524 *$ & -0.522500 & $0.502798 *$ & $\operatorname{LMKI}(-4)$ & -0.060905 & -0.115075 & 0.124780 \\
\hline & $(0.11287)$ & $(0.29114)$ & $(0.56959)$ & & $(0.05822)$ & $(0.15017)$ & $(0.29380)$ \\
\hline \multirow[t]{2}{*}{ LPROD(-2) } & -0.260146 & -0.234710 & 0.360849 & $\operatorname{LMKV}(-1)$ & 0.037888 & -0.322570 & 1.121493 \\
\hline & $(0.20105)$ & $(0.51857)$ & $(1.01454)$ & & $(0.03133)$ & $(0.08080)$ & $(0.15808)$ \\
\hline \multirow[t]{2}{*}{ LPROD(-3) } & -0.092802 & 0.269953 & -0.060926 & $\operatorname{LMKV}(-2)$ & -0.006019 & 0.122957 & -0.195541 \\
\hline & $(0.19855)$ & $(0.51212)$ & $(1.00191)$ & & $(0.03910)$ & $(0.10086)$ & $(0.19732)$ \\
\hline \multirow[t]{2}{*}{ LPROD(-4) } & -0.100232 & -0.024211 & -0.018925 & $\operatorname{LMKV}(-3)$ & 0.014113 & 0.066514 & 0.045906 \\
\hline & $(0.11909)$ & $(0.30718)$ & $(0.60097)$ & & $(0.04034)$ & $(0.10405)$ & $(0.20356)$ \\
\hline \multirow[t]{2}{*}{ LMKI(-1) } & $0.349562 *$ & 0.268241 & $0.977008^{*}$ & $\operatorname{LMKV}(-4)$ & -0.032594 & -0.005717 & 0.193386 \\
\hline & $(0.06326)$ & $(0.16318)$ & $(0.31925)$ & & $(0.02776)$ & $(0.07160)$ & $(0.14008)$ \\
\hline \multirow[t]{2}{*}{$\operatorname{LMKI}(-2)$} & -0.182458 & 0.158436 & -0.313518 & $\mathrm{C}$ & 0.043098 & -0.366852 & 0.457443 \\
\hline & $(0.09340)$ & $(0.24091)$ & $(0.47131)$ & & $(0.04631)$ & $(0.11946)$ & $(0.23371)$ \\
\hline \multirow[t]{2}{*}{ LMKI(-3) } & -0.025526 & 0.131341 & 0.080114 & & & & \\
\hline & $(0.09516)$ & $(0.24544)$ & $(0.48019)$ & & & & \\
\hline \multicolumn{2}{|l|}{$\mathrm{R}^{2}$} & \multicolumn{2}{|c|}{0.997362} & \multicolumn{2}{|c|}{0.978262} & \multicolumn{2}{|r|}{0.705781} \\
\hline \multirow{2}{*}{\multicolumn{2}{|c|}{$\begin{array}{l}\mathrm{R} \text { ajustado } \\
\text { Soma de quadrados dos } \\
\text { resíduos }\end{array}$}} & \multicolumn{2}{|c|}{0.997067} & \multicolumn{2}{|r|}{0.975824} & \multicolumn{2}{|r|}{0.672785} \\
\hline & & \multicolumn{2}{|c|}{0.103201} & \multicolumn{2}{|r|}{0.686599} & \multicolumn{2}{|r|}{2.628006} \\
\hline \multicolumn{2}{|l|}{ Estatística F } & \multicolumn{2}{|c|}{3371.651} & \multicolumn{2}{|r|}{401.2699} & \multicolumn{2}{|r|}{21.38957} \\
\hline \multicolumn{2}{|c|}{ Critério de Akaike } & \multicolumn{2}{|c|}{-4.004020} & \multicolumn{2}{|c|}{-2.108952} & \multicolumn{2}{|r|}{-0.766723} \\
\hline \multicolumn{2}{|c|}{$\begin{array}{l}\text { Critério de Schwarz } \\
\text { Média da Variável }\end{array}$} & \multicolumn{2}{|c|}{-3.702042} & \multicolumn{2}{|c|}{-1.806974} & \multicolumn{2}{|r|}{-0.464744} \\
\hline \multicolumn{2}{|c|}{$\begin{array}{l}\text { Dependente } \\
\text { Desvio da Variável }\end{array}$} & \multicolumn{2}{|c|}{-1.283372} & \multicolumn{2}{|r|}{0.886225} & \multicolumn{2}{|r|}{-1.399650} \\
\hline Dependente & & & 73407 & & .515190 & & 0.273971 \\
\hline
\end{tabular}

Fonte: Dados da Pesquisa.

Com base nos resultados obtidos na Tabela 5, as variáveis mais significativas para explicar o comportamento dos preços ao produtor são LLPROD (-1) e LMKI (-1), ou seja, os preços passados do próprio produtor e do markup da indústria. Para este, apenas o markup do varejo com defasagem de 1 lag foi significativo, e, o sinal encontrado foi negativo. Isso significa que o comportamento do varejo tem efeito inverso 
sobre o comportamento do preço na indústria. No tocante à variável LMKV, as variáveis mais significativas foram a LMKV e LMKI com 1 lag, o que indica que o markup da indústria também afeta o do varejo, assim como o próprio markup do varejo se influencia.

A Tabela 6 reporta os resultados do Teste de Causalidade de Granger realizado após as estimações do modelo VAR. Esse Teste possibilita saber se uma variável (x) causa outra (y), no sentido de Granger, ou seja, se dados os valores passados das variáveis (y) e (x) são úteis para prever os valores de y.

Tabela 6 - Teste de Causalidade de Granger

\begin{tabular}{c|c|c|c|c}
\hline Equação & Excluído & $\mathrm{X}^{2}$ & $\mathrm{gl}$ & Prob \\
\hline LLPROD & LMKI & 42,0097 & 4 & 0,000 \\
LLPROD & LMKV & 3,0957 & 4 & 0,5419 \\
LLPROD & Ambos & 88,7899 & 8 & 0,000 \\
& & & & \\
LMKI & LLPROD & 51,7237 & 4 & 0,000 \\
LMKI & LMKV & 19,6482 & 4 & 0,001 \\
LMKI & Ambos & 94,2769 & 8 & 0,000 \\
& & & & \\
LMKV & LLPROD & 24,9306 & 4 & 0,001 \\
LMKV & LMKI & 19,9952 & 4 & 0,001 \\
LMKV & Ambos & 33,5328 & 8 & 0,000 \\
\hline
\end{tabular}

Fonte: Dados da Pesquisa.

Para cada equação do modelo VAR estimado e para cada variável endógena que não é a variável dependente na referida equação, o teste de Causalidade de Granger testa se os coeficientes estimados (considerando todos os lags) são conjuntamente iguais a zero. Em outras palavras, testa para cada equação do VAR se a variável endógena não causa a variável dependente no sentido de Granger.

Iniciando a análise pela equação com a variável dependente, sendo o log de preços ao produtor, o primeiro teste é verifica se os 4 lags de LMKI são conjuntamente iguais a zero. O valor de probabilidade indica rejeição 
da hipótese nula de que LMKI não causa LLPROD à Granger. Já com relação à variável endógena $L M K V$, não se pode rejeitar a hipótese nula, uma vez que ela não causa LLPROD no sentido de Granger. O terceiro teste está relacionado com a hipótese nula de que os coeficientes dos 4 lags das duas variáveis endógenas são conjuntamente iguais a zero. Como pode ser observado, a hipótese nula é rejeitada. Isso significa que, conjuntamente, o markup do varejo e o da indústria causam LLPROD à Granger. As duas equações restantes possuem a mesma interpretação com relação ao teste de causalidade. Em ambos os casos, seja com relação à variável endógena de forma isolada, seja conjuntamente, rejeitase a hipótese nula. Assim, tanto o preço ao produtor quanto o markup do varejo afetam o markup da indústria, da mesma forma que o preço ao produtor e a margem da indústria afetam o markup do varejo.

\section{Conclusão}

Este trabalho buscou identificar mudanças na estrutura de mercado na cadeia do leite e o efeito sobre seu desempenho entre os anos de $1998 \mathrm{e}$ 2008. O objetivo era tentar mensurar o poder de oligopsônio da indústria processadora e do varejo sobre o produtor, considerando a hipótese de que está ocorrendo um processo de desconcentração no setor.

O setor lácteo passou por mudanças em sua composição (organização), uma vez que um grupo de empresas relativamente menores passou a dominar uma parcela maior de mercado. Os indicadores mostram que a expansão das organizações menores ocorreu em razão da redução da participação de mercado do grupo das empresas líderes, em especial, pela diminuição da parcela de mercado da Parmalat. Essa empresa passou por uma crise financeira e um crescimento desordenado nos últimos anos, de forma que, a partir de 2004, deixou de ocupar a segunda posição, passando para a quarta no ranking.

A análise dos índices de concentração demonstra que o setor está mais desconcentrado porque os grupos menores passaram a atuar no mercado 
em segmentos mais amplos. Já as maiores indústrias, ao longo de dez anos, perderam participação no mercado, principalmente a Parmalat e a Nestlé. Isso confirma o que demonstrou Fernandes (2006) em seu trabalho.

Pelo teste de causalidade de Granger, os markups da indústria e do varejo são responsáveis pelos preços pagos aos produtores. Porém, o markup do varejo e o preço pago ao produtor também causam, no sentido de Granger, o markup da indústria. Por último, tanto produtor quanto indústria causam a Granger o markup do varejo. Em nossa análise, isso sugere que não é possível identificar o exercício de poder de oligopsônio por parte do varejo ou da indústria, sendo mais um indicador de que o setor não possui forte concentração.

\section{Referências}

ALVIM, R. S.; MARTINS, M. C.; MUSTEFAGA, A. P. S. Desempenho da cadeia produtiva do leite no Brasil: visão dos produtores. In: VILELA, D. et al. (Ed.) $\mathbf{O}$ agronegócio do leite e políticas públicas para o seu desenvolvimento sustentável. Juiz de Fora (MG): Embrapa Gado de Leite, 2002. p. 195-204.

CAMPOS, C. K.; PIACENTI, C. A. Agronegócio do leite: Cenário atual e perspectivas. 2007. Disponível em: <http://www.sober.org.br/ palestra/6/1152.pdf.> Acesso em: 04 out. 2009.

CARLTON, D. W.; PERLOFF, J. M. Modern Industrial Organization. 4th ed. Upper Saddle River: Pearson, 2005. 822p.

ELLIOT, G.; ROTHENBERG, T. J.; STOCK, J. H. Efficient tests for an Autoregressive Unit Root. Econometrica, 64, jul. 1996, p. 813-836.

EMBRAPA GADO DE LEITE. Classificação mundial dos principais países produtores de leite de vaca - 2007. Disponível em: <http:// www.cnpgl.embrapa.br/>. Acesso em: 08 set. 2009. 
Fabiano Luiz Alves Barros, João Ricardo Ferreira de Lima \& Rosangela Aparecida Soares Fernandes

FERNANDES, R. A. S., 2006. Mudanças na Estrutura de Mercado láctea e os impactos sobre o desempenho no período de 19972005. Dissertação de Mestrado em Economia Aplicada. Universidade Federal de Viçosa. Viçosa, Minas Gerais. Brasil.

FERNANDES, R. A. S.; SANTOS, C. M. Evolução das Margens de comercialização de leites e queijos no Estado de São Paulo no período de 2000 a 2008. Informações Econômicas (Online), v. 39, p. 1-11, 2009.

GUJARATI, D. Econometria Básica. Rio de Janeiro: Campus, 2006. 812p.

INSTITUTO DE ECONOMIA AGRÍCOLA. Preços pagos aos produtores de leite. Disponível em: <http://www.iea.sp.gov.br/out/banco/ menu.php>. Acesso em: 10 out. 2009.

IPEADATA. Índice Geral de Preço - Disponibilidade Interna. 2009.

Disponvel em: <http://www.ipeadata.gov.br/ipeaweb.dll/ ipeadata? SessionID $=1920008555 \&$ Tick $=1255806773549 \&$ VAR _FUNCAO=Ser_MUso()\&Mod=M>. Acesso em: 10 out. 2009.

MARTIN, S. Industrial Economics - Economics Analysis and Public Policy. 2 ed. New Jersey: Prentice Hall, 1993. 638p.

MUlLER, C. A. S.; SANTOS, J. C.; AGUIAR, D. R. D. Análise de Desempenho na Cadeia Bovina no Estado de São Paulo. In: Sociedade Brasileira de Economia e Sociologia Rural, 44, 2006. Anais do XLIV SOBER. Fortaleza: SOBER, 2006. 14p.

VARIAN, H. Microeconomia: Princípios Básicos. 7 ed. Rio de Janeiro: Editora Campus, 2006. 808p. 


\begin{abstract}
The dairy industry productive chain is among the most important domestic agricultural and cattle raising products, accounting for a significant part of agribusiness. In the last years, this sector has undergone significant structural changes, with increased participation of relatively smaller enterprises and reduced number of larger enterprises in the market. The aim of this work is to understand the dynamics between industry and retail performance and the effect on the prices paid to dairy producers. Specifically, this work aims to calculate the sector's concentration indexes and causality indexes between the prices paid to the producer, the industry markup and the retail prices. Based on price temporal series, a VAR model was estimated and the Granger causality test was carried out. The results obtained reinforce that there was a decrease in concentration in the sector and the causality tests indicate low oligopsony power in retail and industry.
\end{abstract}

Keywords: Market concentration, dairy sector, Granger causality test. 